Quim. Nova, Vol. 35, No. 5, 944-947, 2012

\title{
DESREPLICAÇÃO DE ALCALOIDES APORFÍNICOS E OXOAPORFÍNICOS DE Unonopsis guatterioides POR ESI-IT-MS
}

\author{
Felipe Moura Araújo da Silva*, Hector Henrique Ferreira Koolen, Richardson Alves de Almeida, Afonso Duarte Leão de \\ Souza e Maria Lúcia Belém Pinheiro \\ Departamento de Química, Universidade Federal do Amazonas, Av. Gal. Rodrigo Otávio 3000, 69077-000 Coroado - AM, Brasil \\ Emmanoel Vilaça Costa
}

Departamento de Química, Universidade Federal de Sergipe, Campus Universitário, 49100-000 São Cristóvão - SE, Brasil

Recebido em 14/7/11; aceito em 25/11/11; publicado na web em 20/1/12

\begin{abstract}
DEREPLICATION OF APORPHINE AND OXOAPORPHINE ALKALOIDS FROM Unonopsis guatterioides BY ESI-IT-MS. The dereplication of aporphine and oxoaporphine alkaloids by direct infusion in ESI-IT-MS ${ }^{\mathrm{n}}$ system was applied for alkaloidal fractions of the Unonopsis guatterioides (Annonaceae). Its main advantage over other dereplication methods is the ability to quickly identify substances in complex mixtures without the use of coupled techniques and expensive databases. By only the fragmentation keys and comparison with literature data the aporphine alkaloids anonaine, asimilobine and nornuciferine were identified. The nornuciferine is being reported for the first time in Unonopsis. The oxoaporphine alkaloids liriodenine and lisycamine were identified in the alkaloidal fractions by comparison with the fragmentations of authentic samples.
\end{abstract}

Keywords: alkaloid dereplication; Annonaceae; Unonopsis guatterioides.

\section{INTRODUÇÃO}

A rápida identificação de compostos conhecidos em misturas complexas, uma etapa crucial na busca de novas substâncias bioativas, é um exercício rotineiro nos laboratórios de pesquisa avançados, bem como nas companhias farmacêuticas. Esta prática comumente conhecida como desreplicação faz uso de instrumentação analítica acoplada, como LC-MS e LC-NMR, associada a bancos de dados robustos como a Chapman \& Hall's Dictionary of Natural Products ou o Chemical Abstracts Service's NAPRALERT., Uma vez que estes bancos de dados não são gratuitos, muitas vezes o pesquisador é forçado a montar seu próprio banco de dados e a buscar novas maneiras de fazer a identificação em misturas complexas das mais diversas classes de substâncias encontradas como produtos naturais. Como importante auxílio, existem artigos com sugestões sobre a melhor forma de realizar esta tarefa. ${ }^{2,3}$

O estudo de alcaloides em matriz biológica através de técnicas espectrométricas é uma prática que vem sendo desenvolvida há bastante tempo. ${ }^{4}$ Recentemente foi demonstrado ser possível detectar alcaloides em matriz vegetal por espectrômetro do tipo ion trap mesmo em baixas concentrações. ${ }^{5}$ Estudos feitos com alcaloides aporfínicos purificados em espectrômetro do tipo ion trap demonstraram existir padrões de fragmentação para esta classe de substâncias, servindo de suporte para a caracterização das mesmas. ${ }^{6}$ Essa classe de alcaloides merece atenção especial em função de suas atividades biológicas descritas na literatura. ${ }^{7}$

A família Annonaceae possui 130 gêneros, incluindo o Unonopsis, ainda pouco estudado quimicamente. Apenas 6 entre aproximadamente 50 espécies de Unonopsis registradas ${ }^{8}$ foram submetidas a algum estudo fitoquímico. ${ }^{9-16}$ Diversas investigações revelaram as plantas desse gênero como fontes promissoras de alcaloides aporfínicos e derivados (Tabela 1). Algumas utilizações empíricas de espécies do gênero foram reportadas na literatura, entre elas o uso na composição de veneno para caça e como antifertilizantes, ${ }^{14}$ além de aplicações com fins terapêuticos: índios que vivem ao longo do rio Vaupé adicionam

\footnotetext{
*e-mail: felipesaquarema@bol.com.br
}

folhas secas de $U$. veneficiorum na comida de idosos que sofrem de demência, e folhas pulverizadas de U. stipitata na comida dos que apresentam dificuldade na fala. ${ }^{17}$

Tabela 1. Alcaloides aporfínicos e derivados isolados do gênero Unonopsis

\begin{tabular}{llc}
\hline Espécies & Alcaloides & Refs. \\
\hline U. spectabilis & $\begin{array}{l}\text { Lisicamina, Liriodenina, Onichina, } \\
\text { 6-Hidroxionichina, Ursulina, Macondina, } \\
\text { Isoursulina, Heteropsina e Urabaina }\end{array}$ & $9-10$ \\
U. guatterioides & $\begin{array}{l}\text { Asimilobina, Anonaina, Lisicamina, } \\
\text { Liriodenina e Norushinsunina }\end{array}$ & 11 \\
U. stipitata & Argentinina, Stiptatina e Talictuberine & 11 \\
U. pacifica & $\begin{array}{l}\text { Liriodenina, Norushinsunina, } \\
\text { Norcepharadiona A, Unonopsina e }\end{array}$ & 12 \\
& $\begin{array}{l}\text { Heteropsina } \\
\text { U. butchienii }\end{array}$ & $\begin{array}{l}\text { O-metilmoscatolina, Lisicamina, } \\
\text { Liriodenina e Unonopsina }\end{array}$ \\
& $\begin{array}{l}\text { Asimilobina, Anonaina, Lisicamina, } \\
\text { Uiriodenina, Unonopsina e 5,8-dimetóxi-7- } \\
\text { hidróxi-1-metil-4-azafluoren-9-ona }\end{array}$ & \\
&
\end{tabular}

A espécie $U$.guatterioides apresenta características que a tornam especial dentro do gênero, como a polinização por machos de uma espécie de abelha da tribo Euglossini em vez de coleópteros, ${ }^{18}$ o que é incomum mesmo dentro da família Annonaceae. Considerada a espécie de Unonopsis de mais difícil classificação, ${ }^{8}$ foi a primeira desse gênero estudada fitoquimicamente, por meio de uma prospecção em que se constatou a presença de alcaloides. ${ }^{19} \mathrm{Um}$ ano mais tarde, em um simpósio internacional de química e biologia sobre alcaloides isoquinolínicos, foi relatado o isolamento dos alcaloides aporfínicos asimilobina, anonaina e norunshinsunina, além dos oxoaporfínicos liriodenina e lisicamina de U. guatterioides, ${ }^{11}$ sem indicação da localização destes alcaloides na planta, sendo necessários novos estudos para confirmar a sua constituição. Estas informações químicas e a importância da espécie no gênero justificaram a escolha de $U$. guatterioides como modelo para demonstração da potencialidade 
da técnica aqui aplicada na identificação de alcaloides aporfínicos em misturas complexas. Esta abordagem combina duas ideias existentes no universo da espectrometria de massas em sistema ESIIT-MS: a capacidade de detecção de substâncias mesmo em baixas concentrações em análise por infusão direta $^{5}$ e o conhecimento de padrões de fragmentação, no caso, o de alcaloides aporfínicos. ${ }^{6}$ Os alcaloides oxoaporfínicos, igualmente presentes em Annonaceae, também foram investigados durante as análises por espectrometria de massas no sistema ESI-IT-MS, utilizando-se padrões autênticos para sua confirmação.

\section{PARTE EXPERIMENTAL}

\section{Coleta e obtenção da fração alcaloídica}

O material botânico (folhas, galhos e casca do tronco) foi coletado no Campus da Universidade Federal do Amazonas (UFAM). A espécie foi identificada pelo Prof. Dr. A. C. Webber, Depto. de Biologia do Instituto de Ciências Biológicas (ICB) da UFAM, e uma exsicata foi depositada no Herbário da UFAM sob o número de registro 8249. Após secagem em estufa com circulação de ar a $50{ }^{\circ} \mathrm{C}$ por 2 dias, o material foi triturado em moinho de quatro facas, pesado, pulverizado e macerado durante 3 dias com hexano, seguindo-se a maceração com metanol por igual período. Após a evaporação dos solventes à pressão reduzida, parte dos extratos metanólicos das cascas do tronco $(4,1241$ g), dos galhos $(4,4222 \mathrm{~g})$ e das folhas $(4,2342 \mathrm{~g})$ foi submetida a tratamento ácido-base, conforme a metodologia convencional ${ }^{20}$ para obtenção de alcaloides, fornecendo respectivamente 53,6 mg (1,3\%), $30,9 \mathrm{mg}(0,7 \%)$ e $71,9, \mathrm{mg}(1,7 \%)$ de frações alcaloídicas. De cada fração, separou-se $1 \mathrm{mg}$ para o preparo da solução estoque.

\section{MS}

Os espectros de massas foram adquiridos usando um espectrômetro ion trap LCQ Fleet ${ }^{\mathrm{TM}}$ (Thermo Scientific) equipado com uma fonte de eletrospray, operando no modo positivo e programado para monitorar a faixa de $m / z, 200-400$, adequada aos alcaloides aporfínicos. As soluções estoque foram diluídas a 5 ppm em metanol grau HPLC e aplicadas por inserção direta através da bomba seringa do próprio equipamento. Para aumentar a sensibilidade na análise Tandem, foram adicionados $4 \mu \mathrm{L}$ de ácido acético glacial P.A. (99,8\%) a 10 mL da solução diluída, sendo a concentração final de ácido acético no solvente de $0,04 \%$. Para analisar as frações alcaloídicas das folhas e galhos utilizaram-se os seguintes parâmetros de operação: spray voltage: $5 \mathrm{kV}$; sheath gas: 8 arb; aux gas: 5 arb; sweep gas: 5 arb; capilary temp: $175^{\circ} \mathrm{C}$; capilary voltage: $50 \mathrm{~V}$ (folhas) e $35 \mathrm{~V}$ (galhos); tube lens: $120 \mathrm{~V}$; syringe pump: $8 \mu \mathrm{L} \mathrm{min}{ }^{-1}$. Destes parâmetros, os de sheath gas: 5 arb; capilary voltage: $45 \mathrm{~V}$; tube lens: $115 \mathrm{~V}$ e fluxo de infusão: $10 \mu \mathrm{L} \mathrm{min}{ }^{-1}$ foram os únicos modificados para a análise da fração alcaloídica das cascas do tronco.

\section{LC-APCI-MS}

Para a confirmação da natureza do íon quasi-molecular em $\mathrm{m} / \mathrm{z}$ $282\left([\mathrm{M}+\mathrm{H}]^{+}\right)$foi utilizado um sistema LC-APCI-MS constituído por: um cromatógrafo modelo Surveyor ${ }^{\mathrm{TM}}$ (LC Pump Plus, Autosample Plus) (Thermo Scientific) equipado com uma coluna C18 (Luna), 5 $\mu$, tamanho $150 \times 4,60 \mathrm{~mm}$ (Phenomenex). Utilizou-se na corrida cromatográfica o modo isocrático de eluição com $\mathrm{MeOH} / \mathrm{H}_{2} \mathrm{O}(7: 3)$ como fase móvel durante $15 \mathrm{~min}$; um espectrômetro de massas TSQ ${ }^{\mathrm{TM}}$ Quantum Acess $^{\mathrm{TM}}$ (Thermo Scientific) acoplado ao cromatógrafo através de uma fonte APCI e ajustado para os seguintes parâmetros de operação: discharge current: $3 \mu \mathrm{A}$; vaporizer temperature: 350
${ }^{\circ} \mathrm{C}$; capilary temperature: $270{ }^{\circ} \mathrm{C}$; sheath gas: 30 psi; aux gas: 5 arb. Foi utilizado monitoramento seletivo de íon (SIM), de forma a observar-se nas frações apenas o íon de $m / z, 282[\mathrm{M}+\mathrm{H}]^{+}$. O padrão de nornuciferina e as frações alcaloídicas foram preparados na concentração de $1 \mathrm{mg} \mathrm{mL}^{-1} \mathrm{em}$ metanol grau HPLC. Para a confirmação das demais substâncias, utilizou-se a mesma metodologia descrita acima, alterando-se apenas a substância padrão e, consequentemente, o íon analisado em modo SIM. Os padrões utilizados foram isolados e identificados anteriormente pelos autores..$^{21,22}$

\section{RESULTADOS E DISCUSSÃO}

No espectro de íons totais da fração alcaloídica do extrato dos galhos de $U$. guatterioides foram verificados diversos íons de $\mathrm{m} / z$ par, indicando possíveis alcaloides. O íon mais abundante foi observado, em $m / z, 266\left([\mathrm{M}+\mathrm{H}]^{+}\right)$, cujos fragmentos iônicos obtidos por $\mathrm{MS}^{\mathrm{n}}$ foram caracterizados através da análise das fragmentações chave. ${ }^{6}$ A perda inicial do grupo amina e as sucessivas perdas dos grupos substituintes, $\mathrm{CH}_{2} \mathrm{O}$ e CO (Figura 1a), observadas através da fragmentação até $\mathrm{MS}^{4}$ (Tabela 2), são coerentes para o alcalóide aporfínico anonaína (Figura 2a). A perda inicial do fragmento de massa 17 ou 31 é fundamental para identificar se ao nitrogênio do grupo amina está ligado um hidrogênio ou uma metila, respectivamente. Ainda através da análise das fragmentações chave foi possível identificar os alcaloides aporfínicos asimilobina (Figura 2b) e nornuciferina (Figura 2c) (Figura 1b e 1c). Por sua vez, os alcaloides oxoaporfínicos liriodenina (Figura 2d) e lisicamina (Figura 2e), com íons em $\mathrm{m} / \mathrm{z}, 276$ $\left([\mathrm{M}+\mathrm{H}]^{+}\right)$e $m / z, 292\left([\mathrm{M}+\mathrm{H}]^{+}\right)$, respectivamente, foram identificados por comparação dos respectivos espectros de $\mathrm{MS}^{2}$ com espectros de padrões. Estudos preliminares sobre a fragmentação destes alcaloides revelaram perdas iniciais, que foram associadas à presença de substituintes metoxila e metilenodioxi nas moléculas. Estas perdas iniciais parecem ser características de derivados aporfínicos contendo o átomo de nitrogênio na forma imino e esqueleto de natureza aromática, o que impossibilita a perda de um grupamento amino. Outro pico de massa par ocorreu em $m / z, 272\left([\mathrm{M}+\mathrm{H}]^{+}\right)$. Através da análise de sua fragmentação em $\mathrm{MS}^{\mathrm{n}}$ não foi possível identificá-lo

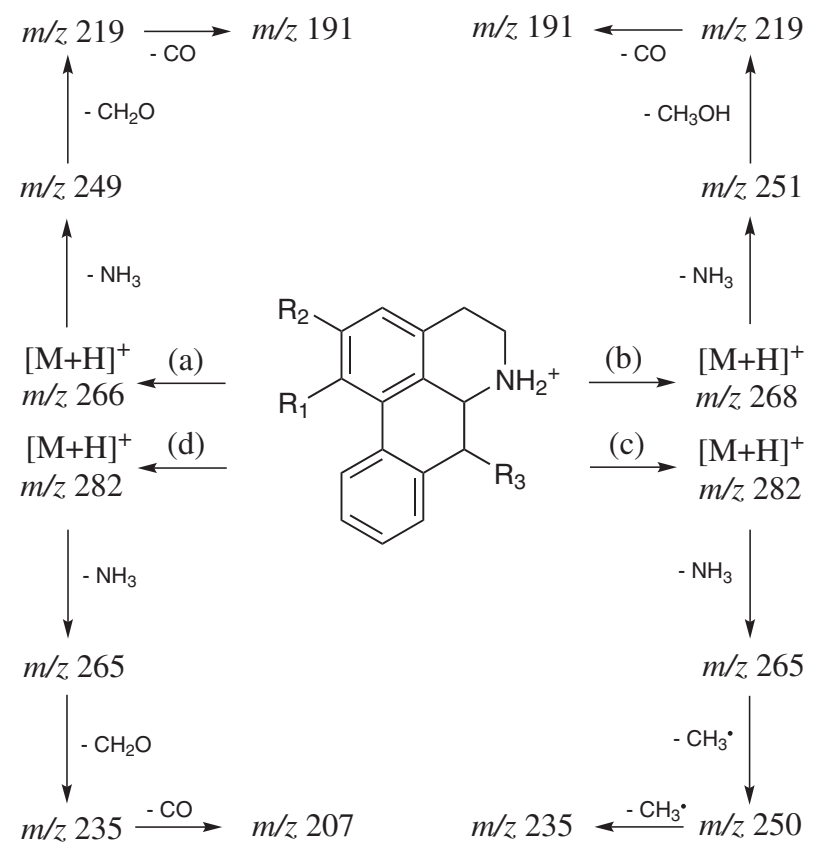

Figura 1. Proposta de fragmentação para a anonaina (a), asimilobina (b), nornuciferina (c) e norushinsunina (d). Para a identificação de $R_{1}-R_{3}$ ver Tabela 1 
como um alcaloide aporfínico. As perdas sucessivas de fragmentos de massa 15 e 18, atribuídos a grupos substituintes da molécula e a perda do fragmento de massa 28, compatível com a presença de um grupo carbonila, possibilitaram supor a ocorrência do alcaloide do tipo azafluorenona, 5,8-dimetóxi-7-hidróxi-1-metil-4-azafluore-9-ona (Figura 2f). Este alcaloide não aporfínico foi recentemente isolado de $U$. lindmanii, ${ }^{16}$ espécie considerada botanicamente próxima de U. guatterioides. $^{8}$<smiles>c1ccc2c(c1)C[C@@H]1Cc3c4cc(c-2c3OCO4)CCN1</smiles>

(a)<smiles>O=C1c2ccccc2-c2c3c(cc4ccnc1c24)OCO3</smiles>

(d)<smiles>COc1c(O)cc2c3c1-c1ccccc1C[C@H]3NCC2</smiles>

(b)

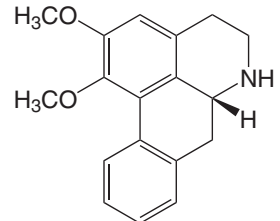

(c)<smiles>COc1cc2ccnc3c2c(c1OC)-c1ccccc1C3=O</smiles>

(e)<smiles>COc1cc(O)c(OC)c2c1-c1nccc(C)c1C2=O</smiles><smiles>O[C@H]1c2ccccc2-c2c3c(cc4c2[C@@H]1NCC4)OCO3</smiles>

(g)
$282\left([\mathrm{M}+\mathrm{H}]^{+}\right)$presente nas folhas e galhos em maior intensidade apresentou um padrão de fragmentação discordante com a estrutura da norushinsunina (Figura 2g). Como observado para a anonaína (Figura 2a), uma ponte metilenodioxi na estrutura deveria assegurar a perda de um fragmento de massa $30\left(\mathrm{CH}_{2} \mathrm{O}\right)$ seguida de um fragmento de massa 28 (CO) (Figura 1d) e, assim, as perdas verificadas de fragmentos de massa 15 em sequência não estariam em concordância com as fragmentações chave. ${ }^{6}$ No entanto, o padrão de fragmentação do íon em $\mathrm{m} / \mathrm{z}, 282\left([\mathrm{M}+\mathrm{H}]^{+}\right)$é coerente com uma estrutura aporfínica contendo um hidrogênio ligado ao átomo de nitrogênio e duas metoxilas ligadas ao sistema aromático (Figura 1c). Com base nas fragmentações chave observadas e com o auxílio de um artigo de revisão desta classe de alcaloides, ${ }^{23}$ chegou-se ao alcaloide nornuciferina (Figura $2 \mathrm{c}$ ) como sendo o possível alcaloide de massa nominal 281. Sua confirmação foi feita através de experimento de LC-APCI-MS (Figura 3) e da comparação das fragmentações do padrão autêntico dessa substância com as fragmentações dos íons de $m / z, 282$ presentes nas frações alcaloídicas dos galhos, cascas do tronco e folhas. No experimento de LC-APCI-MS pode-se observar que sob as mesmas condições cromatográficas e espectrométricas, a detecção da substância de interesse, na forma do íon quasi-molecular em $\mathrm{m} / \mathrm{z} 282\left([\mathrm{M}+\mathrm{H}]^{+}\right)$, ocorre no mesmo tempo de retenção, tanto nas frações quanto no padrão, garantindo assim a confirmação de sua natureza.

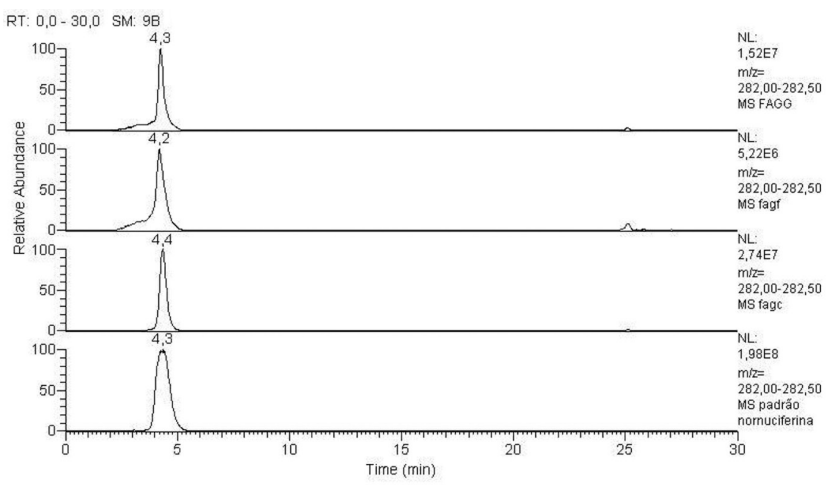

Figura 3. Cromatogramas obtidos por LC-APCI-MS em modo de monitoramento seletivo de íon (SIM) do íon de $\mathrm{m} / z 282\left([\mathrm{M}+\mathrm{H}]^{+}\right)$. De cima para baixo: as frações alcaloídicas dos galhos, folhas e cascas e o padrão de nornuciferina

Analisando a fração alcaloídica do extrato das cascas de $U$. guatterioides foi possível observar a predominância dos alcaloides oxoaporfínicos liriodenina (Figura 2d) e lisicamina (Figura 2e), através da verificação de seus íons quasi-moleculares de massa nominal em $\mathrm{m} / z$ 276 e 292, respectivamente. Dos alcaloides aporfínicos identificados em galhos e folhas, apenas a nornuciferina (Figura 2c) foi identificada na casca. Os alcaloides lisicamina (Figura 2e) e liriodenina (Figura 2f), já relatados na espécie $U$. guatterioides são comuns no gênero Unonopsis (Tabela 1), observando-se que a liriodenina (Figura 2d) é considerada marcador da família Annonaceae. ${ }^{24}$ Os alcaloides aporfínicos identificados nas frações estudadas por ESI-IT-MS ${ }^{\mathrm{n}}$ tiveram seus tempos de retenção comparados com padrões através da análise em sistema LC-APCI-MS, com o espectrômetro operando em modo de monitoramento seletivo de íons (SIM).

\section{CONCLUSÃO}

A desreplicação dos alcaloides aporfínicos de $U$. guatterioides através de infusão direta em sistema ESI-IT-MS ${ }^{n}$ é mais uma demonstração do potencial da espectrometria de massas na simplificação dos estudos de produtos naturais. Em casos específicos essa técnica pode ser suficiente para determinar a composição de uma amostra. através da observação de fragmentação até $\mathrm{MS}^{4}$ (Tabela 2) e análise das fragmentações chave ${ }^{6}$. De fato, o íon quasi-molecular em $\mathrm{m} / z$. 
A aplicação da técnica vai muito além de caracterizar substâncias já conhecidas, servindo também de ferramenta na busca por novas substâncias. A associação do íon quasi-molecular em $m / z, 282\left([\mathrm{M}+\mathrm{H}]^{+}\right)$ com o alcaloide nornuciferina e não com o alcaloide norushinsunina, registrado na literatura para a espécie, contribui para o conhecimento fitoquímico do gênero, uma vez que este alcaloide já isolado de outras anonáceas ainda não havia sido relatado no gênero Unonopsis. Os resultados do presente estudo, incluindo a hipótese da presença de um alcaloide do tipo azafluorenona na planta e a detecção da maioria dos alcaloides aporfínicos isolados anteriormente da espécie $U$. lindmanii (Tabela 1), reforçam o potencial da técnica para a investigação química de plantas desse gênero e ratificam o consenso botânico de similaridade entre esta espécie e $U$. guatterioides.

\section{MATERIAL SUPLEMENTAR}

Os espectros de massas em full scan das frações alcaloídicas dos galhos, folhas e cascas de $U$. guatterioides, os espectros em $\mathrm{MS}^{\mathrm{n}}$ das substâncias caracterizadas no trabalho, assim como os cromatogramas obtidos por LC-APCI-MS para confirmação da natureza dos alcalóides identificados estão disponíveis gratuitamente em http:// quimicanova.sbq.org.br na forma de arquivo PDF.

\section{AGRADECIMENTOS}

Ao Prof. Dr. A. C. Webber pela identificação botânica. Este trabalho teve o suporte financeiro da CAPES, CNPq, FINEP e FAPEAM.

\section{REFERENCIAS}

1. Bobzin, S. C.; Yang, S.; Kasten, T. P.; J. Ind. Microbiol. Biotechnol. 2000, 25, 342 .

2. Fredenhagen, A.; Derrien, C.; Gassmann, E.; J. Nat. Prod. 2005, 68, 385.

3. Corley, D. G.; Durley, R. C.; J. Nat. Prod. 1994, 57, 1484.

4. Roush, R. A.; Cook, R. G.; J. Nat. Prod. 1984, 47,197.

5. Koulman, A.; Tapper, B. A.; Fraser, K.; Cao, M.; Lane, G. A.; Rasmussen, S.; Rapid Commun. Mass Spectrom. 2007, 21, 421.

6. Stévigny, C.; Jiwan, J. L. H.; Rozenberg, R.; Hoffmann, E.; Leclercq, J. Q.; Rapid Commun. Mass Spectrom. 2004, 18, 523.
7. Zanin, S. M. W.; Lordello, A. L. L.; Quim. Nova 2007, 30, 92.

8. Maas, P. J. M.; Westra, L. Y. T.; Vermeer, M.; BLUMEA 2007, 52, 413.

9. Laprévote, O.; Roblot, F.; Hocquemiller, R.; Cavé, A.; J. Nat. Prod. 1987, 50, 984

10. Laprévote, O.; Roblot, F.; Hocquemiller, R.; Cavé, A.; J. Nat. Prod. 1988, 51, 555

11. Guinaudeau, H.; Leboeuf, M.; Cavé, A.; J. Nat. Prod. 1988, 51, 389.

12. Arango, G. J.; Cortes, D.; Cavé, A.; D’ocon, M. P.; An. Quim. C-Org. Bio. 1988, 84, 124.

13. Waechter, A.; Cavé, A.; Hocquemiller, R.; Bories, V. M.; Fournet, A.; Phytotherapy Research 1999, 13, 175.

14. Siqueira, J. M.; Bomm, M. D.; Pereira, F. G.; Garcez, W. S.; Boaventura, M. A. D.; Quim. Nova 1998, 21, 557.

15. Silva, D. B.; Matos, M. F. C.; Nakashita, S. T.; Misu, C. K.; Yoshida, N. C.; Carollo, C. A.; Fabri, J. R.; Miglio, H. S.; Siqueira, J. M.; Quim. Nova 2007, 30, 1809.

16. Yoshida, N. C.; Garcez, W. S.; Siqueira, J. M.; Rodrigues, R. P.; Fabri, J. R.; Resumos da 30 ${ }^{a}$ Reunião Anual da Sociedade Brasileira de Química, Águas de Lindóia, Brasil, 2007.

17. Adams, M.; Gmünder, F.; Hamburger, M.; J. Ethnopharm. 2007, 113, 363.

18. Carvalho, R.; Webber, A. C.; Rev. Bras. Bot. 2000, 23, 421.

19. Forgacs, P.; Jacquemin, H.; Moretti, C.; Provost, J.; Touché, A.; Plant. Med. Phytother. 1983, 17, 22.

20. Costa, E. V.; Pinheiro, M. L. B.; Xavier, C. M.; Silva, J. R. A.; Amaral, A. C.; Souza, A. D. L.; Barison, A.; Campos, F. R.; Ferreira, A. G.; Machado, G. M. C.; Leon, L. L. P.; J. Nat. Prod. 2006, 69, 294.

21. Costa, E. V.; Pinheiro, M. L. B.; Barison, A.; Campos, F. R.; Salvador, M. J.; Maia, B. H. L. N. S.; Cabral, E. C.; Eberlin, N. M.; J. Nat. Prod. 2010, 73, 1183

22. Cruz, P. E. O.; Costa, E. V.; Moraes, V. R. S.; Nogueira, P. C. L.; Vendramin, M. E.; Barison, A.; Ferreira, A. G.; Prata, A. P. N.; Biochem. Syst. Ecol. 2011, 39, 872.

23. Guinaudeau, H.; Leboeuf, M.; Cavé, A.; Lloydia1975, 38, 275.

24. Pinheiro, M. L. B.; Xavier, C. M.; Souza, A. D. L.; Rabelo, D. M.; Batista, C. L.; Batista, R. L.; Costa, E. V.; Campos, F. R.; Barison, A.; Valdez, R. H.; Ueda-Nakamura, T.; Nakamura, C. V.; J. Braz. Chem. Soc. 2009, 20, 1102. 